\title{
Travailler sur des objets en faisant se confronter les points de vue. Regards croisés sur le langage fou : l'exemple des écrits bruts
}

Contrasting points of view. Perspectives on the language of the mad: the example of brut writing

\section{Vincent Capt}

\section{(2) OpenEdition}

\section{Journals}

Édition électronique

URL : http://journals.openedition.org/cel/489

DOI : $10.4000 /$ cel. 489

ISSN : 2262-208X

Éditeur

École du Louvre

Édition imprimée

Date de publication : 1 avril 2014

\section{Référence électronique}

Vincent Capt, «Travailler sur des objets en faisant se confronter les points de vue. Regards croisés sur le langage fou : l'exemple des écrits bruts », Les Cahiers de l'École du Louvre [En ligne], 4 | 2014, mis en ligne le 01 avril 2014, consulté le 19 avril 2019. URL : http://journals.openedition.org/cel/489 ; DOI $10.4000 /$ cel.489

Les Cahiers de l'École du Louvre sont mis à disposition selon les termes de la licence Creative Commons Attribution - Pas d'Utilisation Commerciale - Pas de Modification 4.0 International. 


\section{Cahiers de l'École du Louvre}

recherches en histoire de l'art, histoire des civilisations archéologie, anthropologie et muséologie

\section{Numéro 4. Avril 2014}

Travailler sur des objets

en faisant se confronter les points de vue.

Regards croisés

sur le langage fou : I'exemple des écrits bruts

Vincent Capt

Article disponible en ligne à l'adresse :

http://www.ecoledulouvre/cahiers-de-l'ecole-du-louvre/numero4avril2014/Capt.pdf

Pour citer cet article :

Vincent Capt, «Travailler sur des objets en faisant se confronter les points de vue.

Regards croisés sur le langage fou : l'exemple des écrits bruts », Cahiers de l'École du Louvre, recherches en histoire de l'art, histoire des civilisations, archéologie, anthropologie et muséologie [en ligne] n 4, avril 2014, p. 67 à 75.

\section{(c) creative \\ ㄹ.) $\ominus$ (5)}

(c) École du Louvre

Cet article est mis à disposition selon les termes de la Licence Creative Commons Attribution - Pas d'utilisation commerciale - Pas de modification 3.0 non transposé. 


\section{Cahiers de l'École du Louvre recherches en histoire de l'art, histoire des civilisations archéologie, anthropologie et muséologie \\ Numéro 4. Avril 2014}

Sommaire

Éditorial

p. 1

\section{Étude}

Le musée de Sculpture comparée au prisme de la collection de cartes postales éditées par les frères Neurdein (1904-1915)

Dominique Jarrassé et Emmanuelle Polack

p. $2-20$

Dossier:

Les modalités de la collecte : rapt, troc, marché, fouilles, don...

Et leur impact sur l'objet

Dans collection, il y a collecte...

Introduction de Dominique Jarrassé.

p. $21-23$

Entrer en collection. Pour une ethnographie des gestes et des techniques de collecte

Julien Bondaz

p. $24-32$

De la meilleure façon de constituer une collection. Le cas des émaux «byzantins » de Mikhaill Botkine

Aglaé Achechova.

Edme Antoine Durand (1768-1835) : un bâtisseur de collections

Louise Detrez p. $45-55$

Réunir une documentation pour l'Art Brut : les prospections de Dubuffet dans l'immédiat après-guerre au regard du modèle ethnographique Baptiste Brun p. $56-66$

Travailler sur des objets en faisant se confronter les points de vue : regards croisés sur le langage fou : l'exemple des écrits bruts

Vincent Capt p. $67-75$

D’un regard-pilote à l'écart. L'impact de Dubuffet sur les collectes de l'association L'Aracine

Déborah Couette. p. $76-86$

La cabane éclatée. Morcellement des objets immobiliers apparentés à l'art brut

Roberta Trapani .p. $87-95$ 


\section{Vincent Capt}

\section{Une historicité du « brut de l'art »}

L'art brut intéresse l'analyse de discours parce qu'il a la capacité de faire se croiser les regards portés sur les objets qui s'y trouvent apparentés. Par ailleurs, ces discours conditionnent ces mêmes objets : leur statut varie en fonction de l'appréhension qui en est faite. Les modalités de la collecte sont directement concernées par une telle problématique : dès lors que l'appréhension des objets modèle la réalité même de ceux-ci, la réflexion ne peut se défaire de l'histoire d'un objet, de son parcours institutionnel et de sa mémoire, principalement discursive (saisie non détachée du langage). Dans une perspective comme la nôtre, l'attention portée à la matérialité de l'objet, ici un texte manuscrit, est inséparable du sens qui s'élabore en relation avec certaines discursivités. Alors, suivant Céline Delavaux, "l'art brut ne désigne pas [seulement] des œuvres, mais engendre un complexe discursif qui participe au sens de ces œuvres. D'emblée, l'invention de l'art brut est le symptôme du rapport nécessaire de l'art au langage ${ }^{1}$ ». L'avantage d'une telle saisie consiste en ce qu'elle ne tranche pas entre des lectures visant soit à esthétiser, soit à "pathologiser " les objets soumis à l'analyse.

La réflexion sera conduite à partir d'une lettre de Samuel Daiber ${ }^{2}$ (fig. 1), représentative à bien des égards de l'historicité propre aux œuvres d'art brut : une historicité qui passe du monde de la médecine psychiatrique à celui de l'art. Une historicité fondamentalement moderne, une historicité du dépassement à soi, du devenir autre. C'est ce devenir qui fait le "brut de l'art ", écriture incluse, dont l'efficience se juge principalement par sa capacité à interroger nos classements préétablis : " œuvres d'art ou document cliniques ?3" ", "textes pathologiques ou œuvres d'art ? " $"$.

Un tel devenir conduit, le plus souvent à une difficulté de compréhension de l'art brut ou, plutôt, de saisie de sa spécificité : en somme, " en quoi les œuvres produites par des malades mentaux excèdent[-elles] leur statut de documents cliniques " $"$ ? Cette difficulté est due à un mode d'appréhension qui fige les œuvres dans telle ou telle catégorie préétablie, qui les typologise, les classifie, les range, alors que l'art brut est d'abord une pensée de l'art. Une pensée de l'extension du domaine de l'art développée initialement par Jean Dubuffet dans le contexte de l'immédiat après-guerre, faisant suite à d'autres poétiques de la modernité artistique (pensons à Pablo Picasso avec l'art africain ou à Paul Klee avec les dessins d'enfants), une pensée critique de l'action exercée par l'art sur la société et, plus fondamentalement encore, sur l'ensemble de nos systèmes de lecture préétablis (politique, institutionnel, " genré », ...), en particulier ici le système linguistique même. Une pensée qui n’a de cesse de questionner, de déstabiliser des catégories oppositives, exclusivement distinctes les unes des autres pour signifier (masculin versus féminin, fou/normal, extraordinaire/quotidien,

1. Art brut sans capitales dans ce travail pour ne pas restreindre et figer son appréhension à la Collection homonyme (même si les objets que nous analyserons y sont conservés).

Céline Delavaux, L'Art brut, un fantasme de peintre, Paris, Palette, 2010, p. 316, nous soulignons. 2. Voir Vincent Capt, ÉCRIVAINER La langue morcelée de Samuel Daiber, Lausanne/Gollion, Collection de I'Art Brut/Infolio, 2012, ainsi que Michel Thévoz, « Les missives de Samuel Daiber », $L^{\prime}$ Art Brut, no 11, 1982, pp. 118-133.

3. Béatrice Chemana-Steiner, « Un patrimoine inclassable : œuvres d'art ou documents cliniques ? » dans C. Boulanger et S. Faupin (dir.), Art brut : une avant-garde en moins ?, Paris, L'Improviste, 2011, p. 159.

4. Anouck Cape, «Quand la fée-psychose transforme le pauvre fou en poète », C. Boulanger et

S. Faupin (dir.), op.cit. note 3, p. 32.

5. C. Delavaux, op. cit. note 1 , p. 18 
etc.), instancier les relations et établir des jugements normatifs. L'art brut, tel qu'il a été pensé par le peintre de L'Hourloupe, fait problème à la notion même d'art, institué culturellement. L'art brut part à la découverte de productions qui ne correspondent à rien : non qu'elles n'existent pas, mais parce qu'aucune place assignée préalablement n'est apte à les saisir dans ce qui fait leur spécificité.

La lettre retenue de Samuel Daiber (voir fig. 1) est aujourd'hui indexée aux " écrits bruts ". L'expression revient à l'ancien conservateur de la Collection de l'Art Brut à Lausanne, Michel Thévoz, qui les a appréhendés " par analogie avec [...] l'art brut inventé par Jean Dubuffet $"{ }^{6}$. Cette appellation fait également allusion au titre du recueil éponyme d'écrits asilaires coordonné en 1979 par Thévoz ${ }^{7}$, mais ne s'y restreint pas. Les écrits bruts sont pour la plupart des manuscrits et des œuvres plastiques contenant du texte, produits par des internés asilaires le plus souvent, mais aussi des adeptes du spiritisme et autres isolés marginalisés. Ces écrits sont des lettres, plaidoyers ou journaux intimes, où l'on peut lire la révolte contre l'internement, la solitude et le manque affectif. Leur lecture s'enrichit toujours du rapport à la matérialité linguistique (investissement spécifique du signifiant graphique et/ou phonique) et au support matériel : les auteurs " bruts " travaillent le plus souvent un matériau " pauvre ", ils écrivent sur ce qui est à leur portée, du cahier d'écolier récupéré au morceau de chiffon, en passant par le papier d'emballage, avec un bout de crayon dont il ne reste parfois que la mine.

\section{Mémoire psychiatrique}

La principale discursivité qui a porté les écrits bruts - avant qu'ils ne deviennent tels en tous les cas - est la discursivité psychiatrique. Cette discursivité a impliqué un premier type de "collecte ", réalisé par les médecins psychiatres. Les écrits apparentés à l'art brut proviennent pour la plupart d'archives d'hôpitaux psychiatriques à la suite des investigations menées dans certaines institutions en Suisse et en France par Jean Dubuffet, Michel Thévoz, puis Lucienne Peiry. Après s'être intéressée au corps et à l'anatomie des aliénés, puis à leurs faits et gestes, la psychiatrie s'est penchée sur le langage des internés, au moins à partir de la seconde moitié du XIX siècle, comme lieu d'observation de la sanité ou non des patients. Ce sont donc d'abord les médecins aliénistes, puis les psychiatres, qui ont observé ces écrits, simplement parce qu'ils étaient les premiers, et bien souvent les seuls, à avoir un accès direct aux productions de leurs patients - qui les leur adressaient d'ailleurs le plus souvent, leur demandant libération.

Anouck $\mathrm{Cape}^{8}$ ajoute à ces remarques que la quantité d'écrits asilaires a toujours été importante : les patients psychiatriques écrivent beaucoup durant leur enfermement. Cependant, les textes à disposition représentent une infime partie de productions initialement très importantes quantitativement. La plupart de ces écrits étaient conservés au sein même des dossiers médicaux consacrés aux patients, parmi des données médicales, comme c'est le cas de la lettre de Samuel Daiber, avec la mention hétérogène de la date " 20/X. 67 " servant à classer le texte. On peut imaginer qu'une sélection minimalement représentative des troubles langagiers observés suffisait au dossier pour illustrer tel ou tel trouble. Les documents étaient principalement retenus pour leur valeur diagnostique. Il est alors légitime de penser que la majeure partie des textes asilaires désormais apparentés à l'art brut - par exemple ceux de Daiber, rédigés au moins entre 1954 et 1967, au nombre de dix-huit finalement -, a tout simplement disparu.

Béatrice Chemana-Steiner donne pour sa part à cette relative rareté des raisons propres à la logistique des diverses administrations asilaires, plus ou moins bien organisée alors. Elle indique que la plupart de ces productions n'entraient dans aucun classement prévu : "errantes dans l'institution " , défaites de statut

6. Michel Thévoz, Le Langage de la rupture, Paris, PUF, 1978, p. 10.

7. M. Thévoz, Écrits bruts, Paris, PUF, 1979.

8. A. Cape, Les Frontières du délire : écrivains et fous au temps des Avant-gardes, Paris, Honoré Champion, 2011.

9. B. Chemana-Steiner, op. cit. note 3, p. 161. 
administratif, elles ont le plus souvent été jetées ou détruites. Ces écrits sont de véritables " rescapés de l'oubli » ${ }^{10}$. Parce qu'ils ne correspondaient à rien, ces écrits n’ont pas trouvé place : nomades donc évacués.

Dans le regard psychiatrique, le langage desdits aliénés a été conservé à titre d'échantillon afin d'établir une première symptomatologie des troubles langagiers, décrits et classés en autant de symptômes distinctifs pour former le système des maladies mentales. Dans ce cas, le langage a été transféré vers le système des pathologies pour signifier comme symptôme ${ }^{11}$. Ce déplacement a été soutenu par l'idéologie de la transparence du langage : les signes linguistiques ont en effet permis de déchiffrer le fonctionnement du cerveau. Les idées sont alors révélées par le langage : littéralement, le regard psychiatrique y lit dans la pensée de l'aliéné. C'est sur ce modèle de la transparence qu'est bâti l'entier de l'étiologie de la médecine mentale : les causes des pathologies ont toujours été rétablies à partir d'une manifestation sémiologique (comme indiqué supra, d'abord l'anatomie de l'aliéné, son comportement, puis son langage). Dans tous les cas, la manifestation reflète une lésion, elle vaut comme « miroir du dedans $»^{12}$.

C'est avec ce modèle que la lecture aliéniste a appréhendé le langage des internés comme écart, pathologique. Le protocole d'observation psychiatrique du langage a ainsi soutenu la logique du signe et, suivant Juan Rigoli ${ }^{13}$, s'est aussi trouvé redevable de la rhétorique - judiciaire principalement, assurant les bases de la médecine légale -, soucieuse de fixer des normes, sinon des lois, à partir desquelles les déviances peuvent être signalées, puis mesurées : aux yeux du " tribunal médical ", les écarts ont ainsi une valeur différentielle, contrastive. Le langage des aliénés signe alors leur folie, l'essence même de celle-ci. Sortant des conventions, il garantit une vérification objective de tel ou tel trouble. La collecte de ces écrits en milieu psychiatrique est donc elle-même redevable du schéma du signe linguistique. C'est l'idée forte que soutient également Michel Pierssens : "Nous disons que cette folie, et depuis toujours (depuis Cratyle), c'est le signe qui la rend possible ${ }^{14} »$.

Considérées à partir du signe, certaines productions dont la rationalité ne serait pas calquée sur sa logique biface - articulation arbitraire, mais nécessaire, entre signifiant/signifié - pour signifier et qui impliqueraient d'autres modes de lecture pour valoir ne peuvent par opposition qu'être folles. Dans cette perspective, la folie n'est possible qu'à l'intérieur d'un savoir, relativement à celui-ci - en l'occurrence celui que fait voir le signe linguistique. En retour, une telle explication montre notre folie, conventionnelle : celle de réduire la saisie du langage à sa représentation par le signe seul. Elle fait voir que nous sommes tous "fous du signe ". Avec la psychiatrie et l'équivalence qu'elle pose entre signe/ symptôme, nous sommes tous "malades " d'une conception du langage : de façon symptomatique, nous peinons pour la plupart à voir et entendre ce dernier autrement que dans sa valeur indicielle.

C'est pourtant ce que requiert le langage quand celui-ci concerne l'art, quand il active une poétique, la sienne propre, qui assoit l'autre écoute du langage requise par les écrits asilaires devenus " bruts ». L'idée n'est pas nouvelle. Certains ont tenté de faire valoir les écrits asilaires autrement. Par rapprochement avec la poésie tout d'abord (dès 1860, les titres de thèses de médecine sont éclairants à ce sujet) et, presque simultanément, avec la littérature. C'est ce qu'indique Anouck Cape :

«Perçus, depuis la seconde moitié du XIXe siècle, comme du matériel clinique de première importance quant à l'établissement d'un diagnostic, ces textes, collectés, retranscrits, cités et commentés par les psychiatres dans diverses publications spécialisées ont peu à peu rencontré un autre public, étranger à

10. B. Chemana-Steiner, op. cit. note 3, p. 159.

11. A ce propos, il est intéressant de rappeler que dans les dictionnaires contemporains de langue, le terme «symptôme » a pour synonyme celui de signe. Notre conception du langage porte ainsi la marque, sinon la mémoire du symptôme.

12. Marcel Gauchet, « Personne, individu, sujet, personnalité », M. Gauchet et J.-C. Quentel (dir.), Histoire du sujet et théorie de la personne, Rennes, PUR, 2009, p. 19.

13. Juan Rigoli, Lire le délire. Aliénisme, rhétorique et littérature en France au XIXe siècle, Paris, Fayard, 2001.

14. Michel Pierssens, « Le Signe et sa folie », Romantisme, vol. 9, nos 25-26, 1979, p. 51. 
la sphère médicale, qui leur a conféré le statut d'œuvres littéraires - les fous du XIX ${ }^{e}$ siècle venant ainsi hanter l'imaginaire du $\mathrm{XX}^{\mathrm{e} 15}$."

Dans tous les cas, l'approche renouvelée de certains écrits de l'enfermement requiert de délire, c'est-à-dire étymologiquement (delirare), "sortir du sillon " de nos habitudes de lecture : du point de vue linguistique, faire perdre au signe sa seule raison de signe pour lui en faire gagner une autre (de raison) et ouvrir en son sein à une altérité qui le fasse valoir. Suivant cette perspective, fondée à partir des derniers travaux d'Émile Benveniste ${ }^{16}$ et développée dans le sillon de l'œuvre d'Henri Meschonnic ${ }^{17}$ et sa théorie du poème, l'affolement est le principe de l'art. Nous développerons ce point infra. Retenons pour l'heure simplement la proposition de Gérard Dessons, pour qui « [1] e poème est le nom de la folie dans le langage $»^{18}$.

\section{L'aventure d'une collecte : les lettres de Samuel Daiber (1902-1983)}

Les lettres de Samuel Daiber ont intégré les archives de la Collection de l'Art Brut en deux temps. Un premier ensemble de treize textes a été acquis par Michel Thévoz vers 1978, avec l'appui du Docteur Ralph Winteler, dont Daiber était un des patients à Perreux, dans le canton de Neuchâtel, en Suisse romande. À la suite de notre recherche ${ }^{19}$ et à nouveau grâce à la collaboration de ce même médecin psychiatre, le musée lausannois a acquis en 2008 cinq nouvelles lettres, dont celle que nous retenons ici (fig. 1), rédigée au stylo-bille sur une demi page de papier ligné, encadrée par un ruban adhésif.

Figure 1

Samuel Daiber, Monsieur Albbagli Hospice Perreux H. Suisse 20 octobre 1967

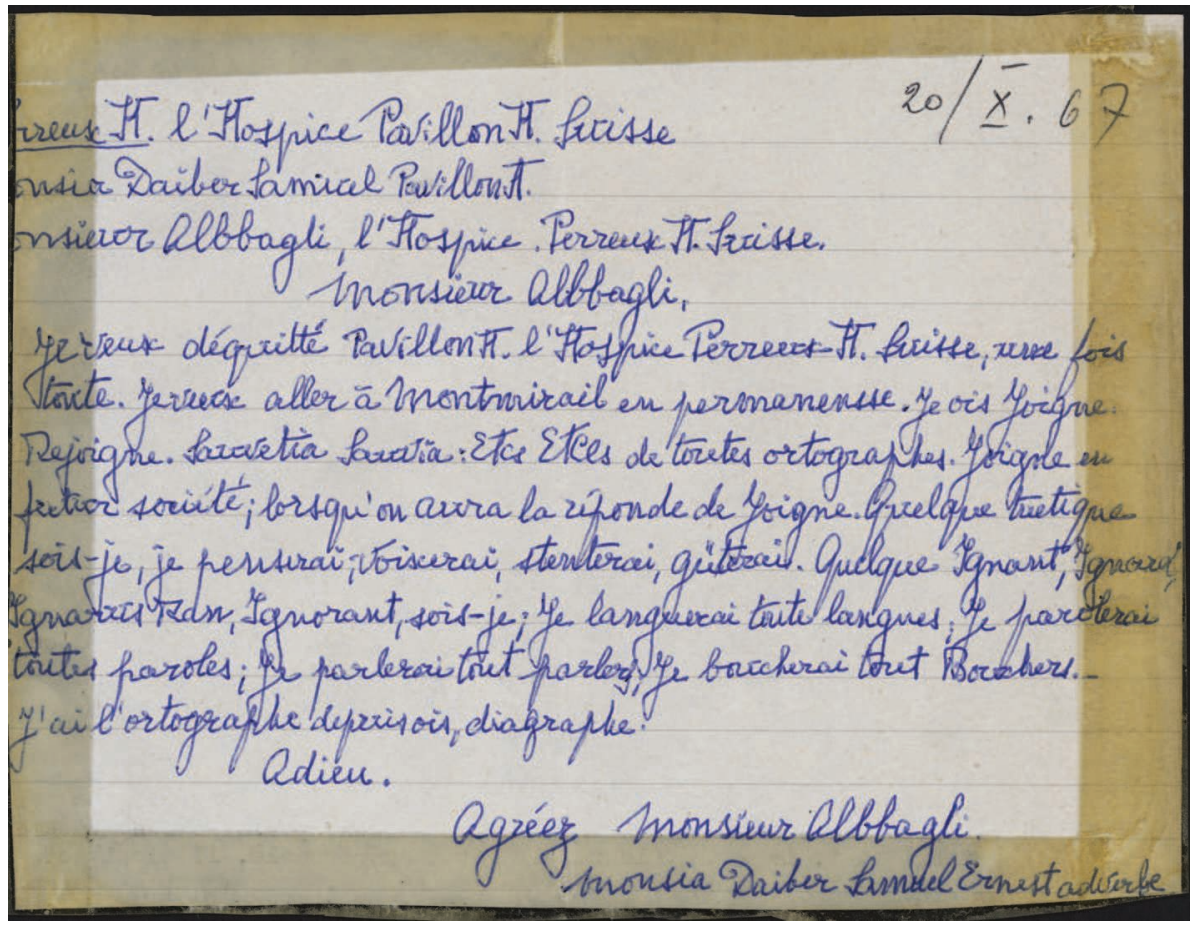

Ces cinq lettres étaient conservées jusque-là dans les dossiers médicaux de l'Hôpital psychiatrique de Neuchâtel. Pour les deux collectes (1978 et 2008),

15. A. Cape, « De l'aliénisme à la littérature d'avant-garde ou les ambiguïtés d'une consécration : petite histoire des écrits de fous $\gg$, Romantisme, n 141/3, 2008, p. 66.

16. Émile Benveniste, Baudelaire, présentation et transcription de Ch. Laplantine, Limoges, Lambert-Lucas, 2011.

17. Henri Meschonnic, Langage histoire, une même théorie, Paris, Verdier, 2012.

18. Gérard Dessons, La Manière folle. Essai sur la manie littéraire et artistique, Paris, Manucius, coll. « Le marteau sans maître », 2010.

19. V. Capt, op. cit. note 2. 
le rôle d'un intermédiaire a été prépondérant ${ }^{20}$. Sachant que les missives de Samuel Daiber ne prétendaient à aucune diffusion artistique, qu'elles étaient tenues anonymes en raison du secret médical, l'intervention tierce d'acteurs provenant directement des deux institutions respectives (médecin psychiatre et conservateur) et celle combinée du chercheur - ce dernier intégrant alors la démarche de la collecte - a été primordiale pour transférer ces lettres dans un musée artistique.

L'on atteint ce que Nathalie Heinich ${ }^{21}$ a qualifié de " quatrième génération de chercheur ", dont la relation avec l'objet d'étude est spécifique dans la mesure où elle ne se défait pas d'une dimension critique avec le cadre même des valeurs attribuées aux discours et aux objets analysés. Cette relation ne réduit pas le chercheur au seul rôle d'observateur plus ou moins coupé des réalités sociales et langagières qu'il décrit. Elle indique au contraire qu'il peut prendre plus ou moins activement part à l'élaboration de son objet d'étude. Ce type de relation indique en retour que le corpus n'aurait jamais été donné à lire sans notre intervention. Notre lecture constitue en ce sens un geste fort : le texte retenu de Daiber (voir fig. 1) n'a jamais été publié, il n'est donné à voir que parce qu'il est porté par un discours - le nôtre - avec lequel est établi un dialogue. Ces quelques indications ouvrent à une perspective constructiviste, collecte liée à un geste de constitution, non axiologique, mais engagée du point de vue critique par la déconstruction. Il ne s'agit pas d'établir un jugement de valeur, mais de tenir compte des évaluations établies à propos de l'objet au cœur de la réflexion, dont voici une transcription de travail :

Perreux H. l'Hospice Pavillon H. Suisse

Monsieur Daiber Samuel Pavillon H.

Monsieur Albbagli, l'Hospice. Perreux H. Suisse

Monsieur Albbagli,

Je veux déquitté Pavillon H. l'Hospice Perreux H. Suisse, une fois toute. Je veux aller à Montmirail en permanensse. Je vis Joigne Rejoigne. Sauvetia Sauvia : Etcs Etces de toutes ortographes. Joigne en fection société ; lorsqu'on aura la réponde de Joigne. Quelque tuetique sois-je, je penserai ; voixerai, stenterai, güterai. Quelque Ignant, Ignard, Ignarris Ran, Ignorant, sois-je ; Je languerai toute langues ; Je parolerai toutes paroles ; Je parlerai tout parlers; Je boucherai tout Bouchers.

J'ai l'orthographe depérisois, diagraphe.

Adieu.

Agréez Monsieur Albbagli.

Monsia Daiber Samuel Ernest adverbe

Cette lettre a été écrite en novembre 1967. Elle est adressée à un membre de l'équipe médicale qui officiait alors à Perreux. Outre la transcription que nous en proposons, elle bénéficie de deux états de texte. Il faut savoir en effet qu’avant son transfert en 2008 à la Collection de l'Art Brut, le ruban adhésif était encore collé à une feuille de papier A4 émanant de l'administration de l'Hôpital psychiatrique cantonal de Neuchâtele ${ }^{22}$, dont il ne reste désormais qu’une photocopie (fig. 2) :

20. D'autres figures d'intermédiaire existent évidemment dans le contexte de l'art brut. C'est le cas de certains agents de diverses prospections. Cette dernière est particulièrement développée dans le contexte de l'art brut, via la figure de «l'amateur passionné » suivant une expression de Laurent Danchin. Pour cette figure, il importe précisément de découvrir des productions tenues jusque-là cachées ou secrètes. Comme indiqué supra, cette figure d'un tiers, d'un relayeur est primordiale pour ce qui concerne la collecte des œuvres apparentées à l'art brut. Elle pourrait par exemple être attribuée à Pascal Rigeade, responsable du musée Création Franche, qui dit lui-même être un « passeur ».

21. Nathalie Heinich, La Sociologie de l'art, Paris, La Découverte, 2004 [2001].

22. Se reporter aux inscriptions en capitales sur l'état antérieur du texte. 
En dessous du collage figure sur cette fiche une remarque de type diagnostic psychiatrique, rédigée par un membre du corps médical de Perreux :

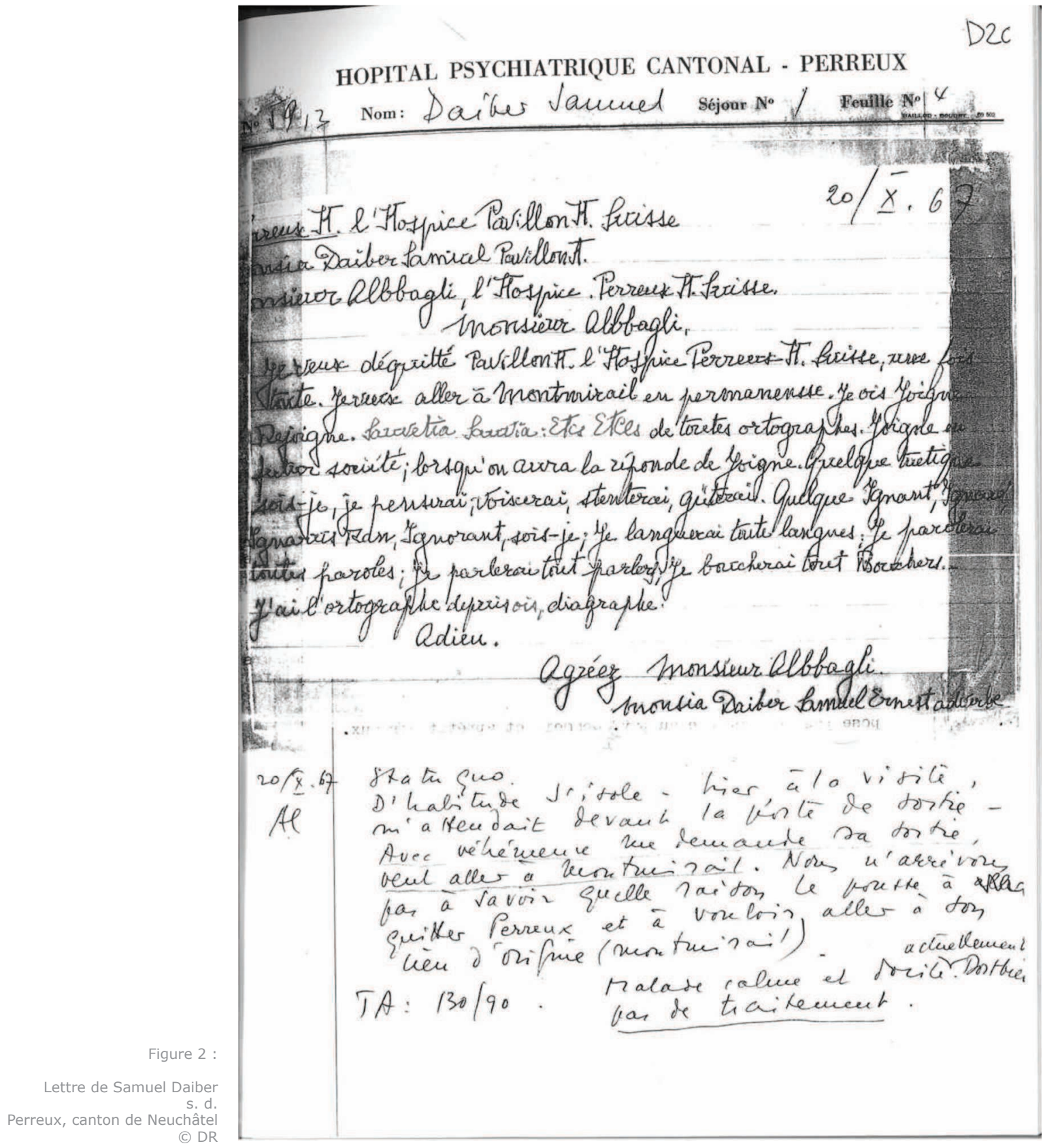

20/X. 67 Statu quo

Al. D'habitude s'isole - hier, à la visite, m'attendait devant la porte de sortie. Avec véhémence me demande sa sortie, veut aller à Montmirail. Nous n'arrivons pas à savoir quelle raison le pousse à alter quitter Perreux et à vouloir aller à son lieu d'origine (Montmirail).

TA : 130/80. Malade calme et docile. /actuellement/ Dort bien pas de traitement

Parmi les dix-huit missives de Samuel Daiber désormais archivées à la Collection de l'Art Brut, celle-ci est la seule dont la matérialité a été modifiée après son entrée au musée lausannois : ce texte est désormais détaché du support sur lequel Daiber l'avait collé (la feuille administrative de l'hospice de Perreux). Seule a été conservée la demi-page lignée et encadrée par son ruban adhésif, coupant le texte d'un pan de son historicité. Ces deux états reflètent bien les différents regards qui ont été portés sur ce manuscrit. Ils montrent surtout la difficulté à ne pas 
enfermer, ici un objet textuel, dans telle ou telle nomenclature, la difficulté à sortir des grilles préétablies de lecture, exclusives, pour signifier.

Lorsque Michel Thévoz s'est penché une première fois sur les écrits de Samuel Daiber dans le recueil Écrits bruts, il avait désigné l'épistolier et avait transcrit les mentions de son nom dans les lettres par l'indication du seul prénom "Samuel ", suivi de l'initiale de son patronyme "D. ", afin de ne pas communiquer son identité juridique complète. Or « Samuel D. » est initialement une discursivité carcérale, ici psychiatrique. Elle rappelle l'anonymat dans lequel étaient plongés les internés asilaires ${ }^{23}$. La désignation incomplète des épistoliers ne revient cependant pas à Thévoz lui-même. Ce sont en effet les directeurs d'institution psychiatrique qui ont imposé le maintien de l'anonymat de certains épistoliers, comme contrainte d'édition de l'ouvrage. Dans ce cas, il y a bien superposition de deux discursivités, psychiatrique d'une part et artistique ou, davantage, socio-esthétique de l'autre.

Cette superposition ne doit pas masquer le fait que les regards portés ne peuvent se recouvrir et qu'ils sont même antithétiques : tandis que certains ont vu des symptômes à enfermer, d'autres ont lu, à des époques différentes, de la poésie ou une pratique libératrice et contestataire. Chaque lecture est située : dans une perspective antipsychiatrique et sociocritique, Michel Thévoz a ainsi insisté sur la productivité lexicale défaite des contraintes communicationnelles caractéristiques des écrits de Daiber, qui seule a justifié de les extraire du circuit psychiatrique pour les diffuser dans le domaine culturel et les faire connaître au public du musée.

Du point de vue d'une poétique, la langue de Samuel Daiber est identifiable dans tous les courriers que celui-ci a envoyés : on la reconnaît et on la nomme à travers un nom de sujet : "du Daiber ", subjectivé dans et par la valse morphologique des entités lexicales sans équivalent préalable dans la langue, tels "voixerai ", « languerai » ou " parolerai », qui cumulent les figures de construction morphématique (conversion et flexion). D'abord, les substantifs « voix », «langue » et " parole " changent de nature grammaticale en raison de la suppression de tout article qui les précèderait ; puis ces néologismes sont conjugués - ajout de la désinence de la première personne du futur - et finissent d'acquérir un statut verbal. Plus radicalement, la subjectivation des unités lexicales atteint la base de certains lexèmes, comme c'est le cas de "stenterai " ou " güterai ", qui ne conservent en finale que les morphèmes grammaticaux mentionnés supra. Le radical de ces mots ne contient pas d'unité linguistique dotée d'un sens. Les unités graphiques impliquent alors de se libérer du schéma morphologique de la langue et peuvent être abordés comme valeur : défaites de la logique du sens préétabli linguistiquement, elles s'inventent et signifient dans le langage en tant que sujet.

Caractérisée par son potentiel exploratoire, l'énonciation de Daiber n'emprunte pas uniquement la voie de mécanismes de suffixation, redistribuant les morphèmes, ici grammaticaux. Elle n'a pas exclusivement trait à des combinaisons morphématiques plus ou moins inédites; elle a la capacité de ne pas conserver les atomes sémantiques de la langue pour forger des unités graphiques spécifiques. Certains mots agissent en retour sur les conventions de la langue dans la mesure où ils peuvent signifier indépendamment du système morphologique ou, en tous les cas, en l'interrogeant et en l'affolant. Ces mots sont donc actifs dans le langage. Ils sont le fruit d'un sujet critique, un sujet qui rend sa spécificité artistique à la folie : l'énonciation de Daiber a "affolé le morphème ». En ce sens, une poétique du délire n'a rien à voir avec une symptomatologie de celui-ci. Son efficience ne concerne pas l'identification générique d'une pathologie préalablement répertoriée, mais la reconnaissance d'une énonciation individuante, à découvrir toujours. C'est ce régime de la subjectivité qui permet de déplacer la folie du champ de la psychologie, entendue au sens large, vers celui du langage. C'est ce

23. Le procédé n'est pas systématique dans Écrits bruts. Ainsi, le nom d'« Henri Müller », par exemple, est conservé comme tel (certainement parce que cet auteur était déjà apparenté à l'art brut, préalablement au recueil d'écrits. À l'inverse, Gaston Marchal-Moreau est nommé via la contraction «Marmor », auto-attribuée, tandis que «Sylvain », pour Sylvain Lecoq, ne conserve que le prénom. Dans son article de 1982 cité note 2, Thévoz mentionne le prénom et le nom complets de l'épistolier. 
déplacement qui fait la spécificité des œuvres apparentées à l'art brut, passant de la manie à la manière ${ }^{24}$.

\section{La manie ou la manière : vers une poétique de la folie}

La manie est une discursivité psychiatrique, qui fait voir par exemple les obsessions, le martèlement ou la stéréotypie, la multiplication ou la dissociation. Elle fait valoir des symptômes. Partant, la discursivité psychiatrique ne peut pas informer de la spécificité artistique. La manière, elle, provenant notamment de mania, signifiant "délire ", concerne également la folie, mais une folie spécifique, ni pathologique ni incarnée. La manière est le nom de la folie dans le langage. Elle est le principe actif de l'art, à condition que celui-ci ne se laisse pas rationaliser exclusivement par une seule grille préexistante. Pour la langue de Daiber, nous avons vu en quoi le système des morphèmes de la langue française pouvait être affolé. Avec le point de vue de la manière, l'art concerne une poétique, une invention et un dépassement individuant, non une esthétique, du beau. Une poétique n'est autre que l'exploration de ce qui échappe, de ce qui se dérobe à un système préétabli de lecture, de ce qui fait problème, de ce qui interroge nos conventions. En un mot, de ce qui affole, de ce qui nous affole - nos conventions. Lart alors rend fou.

Cette association de la folie à l'art est explicite chez l'inventeur de l'art brut. Dans une lettre pour l'heure inédite ${ }^{25}$ adressée en janvier 1947 au peintre René Auberjonois, Jean Dubuffet indique : " [j]e pense qu'il n'y a pas d'art des fous. L'art des fous est le même que l'art des sains d'esprit. Fou sans doute toujours : l'art est toujours fou ". Ailleurs, le peintre havrais soutient la même idée : " [1]a création d'art, où qu'elle apparaisse, est toujours dans tous les cas pathologique ${ }^{26}$ ". Si l'art est malade, c'est parce qu'il est l'autre du langage, l'autre au sein du langage. Parce que l'art signe l'aliénation du langage, au sens où il trace son altération, son devenir étranger. Cet affolement garantit l'efficience critique des œuvres «brutes ». Il a permis à Dubuffet d'inclure dans l'art ce qui lui échappait, ce qui était majoritairement restreint à une autre folie : la manie. Cet affolement, moderne, a rendu possible une poétique du brut, qui conjoint pensée de l'art et pratique de la collecte.

En définitive, nous nous sommes intéressé à une collecte parce que celle-ci a pu éclairer en amont les points de vue qui l'ont fait valoir. Ici, manie et manière ne signifient pas ensemble parce qu'elles ne le peuvent pas : elles ne savent pas voir les mêmes formes, elles activent des valeurs qui leur sont chaque fois propres. Les collectes des productions apparentées à l'art brut effectuées dans des institutions psychiatriques passent ainsi d'une folie à une autre. L'historicité des œuvres d'art brut est caractérisée par ce double mouvement : elle implique dans un premier temps de tenir compte de la manie, la maladie mentale, puis, dans un second, de la dépasser. Une poétique n'ignore pas la manie : à ses yeux, cette " première folie ", médicale, est un moment de l'historicité de l'objet qui n'a cependant plus de raison de valoir dans le monde de l'art. Une poétique exige d'en explorer une nouvelle (de folie). La folie de l'art. La manière. Celle que fait finalement voir l'art brut, par-delà tel ou tel délire pathologique. Dans ces conditions, et comme le synthétise Céline Delavaux : « [l]'art brut décrit non pas la folie de l'artiste, mais celle de l'œuvre, celle de l'art ${ }^{27} »$.

\footnotetext{
24. Sur ce couple notionnel, se reporter à Gérard Dessons, L'Art et la manière. Art, langage et littérature, Paris, Honoré Champion, 2004 et G. Dessons, 2010, op. cit. note 18.

25. Nous soulignons. Lettre conservée au Centre de Recherche sur les Lettres Romandes (CRLR), à Lausanne. Elle sera reproduite dans le livre en préparation dirigé par Daniel Maggetti consacré à la correspondance entre Jean Dubuffet et certains acteurs suisses du monde de l'art et de la littérature (René Auberjonois, Paul Budry, Charles-Albert Cingria et Henry-Louis Mermod).

26. Jean Dubuffet, Prospectus et tous écrits suivants, t. 1, Paris, Gallimard, 1967, p. 456.

27. Céline Delavaux, « L'Art brut n'est pas I"art des fous" », Actes 1 du séminaire sur l'art brut, Paris, abcd \& CIPH, 2012, p. 55.
} 


\section{L'auteur}

Maître-assistant en linguistique à l'Université de Lausanne, Vincent Capt est Docteur en langue et littérature françaises (Lausanne/Paris 8). Il est l'un des membres fondateurs du Collectif de réflexion autour de l'Art Brut (CrAB). Ses travaux portent sur la théorie du langage (énonciation, poème, discours) et sont articulés autour du thème de la folie (écrits bruts, Michaux, Artaud...). Son dernier ouvrage a paru en 2013 : Poétique des écrits bruts. De l'aliéné vers l'autre de la langue. 